\title{
Palatonasal Recess on Medial Wall of the Maxillary Sinus and Clinical Implications for Sinus Augmentation via Lateral Window Approach
}

\author{
Hsun-Liang Chan, * Alberto Monje, * Fernando Suarez, ${ }^{*}$ Erika Benavides, ${ }^{\dagger}$ and Hom-Lay Wang*
}

Background: Anatomic variations of the maxillary sinus determine the degree of difficulty in performing sinus augmentation. Whereas some variations, e.g., the septum and morphology of the sinus, have been extensively studied, the structure of the medial wall has never been investigated. The aims of this study are to measure the location and angulation of the palatonasal recess (PNR) on the medial wall and identify risk sites that are related to the shape of the PNR.

Methods: Cone-beam computed tomography (CBCT) scans were screened from the University of Michigan School of Dentistry database. Edentulous sites with $<10 \mathrm{~mm}$ between the floor of the maxillary sinus and the alveolar crest were selected. The residual ridge height $(\mathrm{RH})$, the distance between the PNR and the alveolar crest, and the angulation of the PNR were measured on the selected sagittal planes. The percentage of sites (risk sites) with recesses that were $<90^{\circ}$ and $<15 \mathrm{~mm}$ from the alveolar crest was calculated. The PNR location and angulation were compared among premolar and molar edentulous sites.

Results: Two hundred seventy-four sites were studied. The mean \pm SE PNR location was $14.2 \pm 2.8 \mathrm{~mm}, 13.1 \pm$ $2.2 \mathrm{~mm}$, and $12.5 \pm 2.5 \mathrm{~mm}$ for the second premolar, first molar, and second molar sites, respectively, with significant differences between the second premolar and second molar sites. The mean PNR angulation was $109.8^{\circ} \pm 25.3^{\circ}$, $121.6^{\circ} \pm 22.1^{\circ}$, and $144.9^{\circ} \pm 23.1^{\circ}$ in the corresponding sites, with significant differences among the site groups. The respective percentages of risk sites were $15 \%, 8.2 \%$, and $2.4 \%$ in the second premolar, first molar, and second molar sites.

Conclusions: Maxillary sinuses with acute-angled PNRs might present a challenge for performing sinus augmentation. Therefore, this anatomic structure should be carefully evaluated. J Periodontol 2013;84:1087-1093.

\section{KEY WORDS}

Alveolar ridge augmentation; dental implants; maxillary sinus/surgery; paranasal sinus diseases/complications; oral surgical procedures, preprosthetic.

\footnotetext{
* Graduate Periodontics, Department of Periodontics and Oral Medicine, University of Michigan School of Dentistry, Ann Arbor, MI.

$\dagger$ Division of Oral Pathology/Medicine/Radiology, Department of Periodontics and Oral Medicine, University of Michigan School of Dentistry.
}

$\mathrm{R}$ estoration of the posterior edentulous maxilla by dental implants often presents a challenge because of inadequate quality and quantity of alveolar bone. After the tooth is lost, both the alveolar bone and the floor of the maxillary sinus increase the remodeling process, resulting in alveolar bone resorption and sinus pneumatization. Several treatment strategies have been developed to overcome anatomic deficiencies; among them is the sinus augmentation via the lateral window (SALW) approach. Described by Boyne and James ${ }^{1}$ and Tatum Jr., ${ }^{2}$ SALW aims to increase the vertical bone height by elevating the sinus floor. According to the original descriptions, an osteotomy was made on the maxillary lateral wall, followed by elevation of the Schneiderian membrane and implantation of bone grafts in the space that was created. ${ }^{1,2}$ The original technique has been subsequently modified by using different instruments, such as the piezoelectric machine, ${ }^{3}$ the balloon, ${ }^{4}$ and the biologic agent. ${ }^{5}$ SALW has been proven to be predictable in terms of amount of bone gain and survival of implants placed in grafted sinuses. 6,7

One of the prerequisites for a successful SALW procedure is prudent management of the Schneiderian membrane. Perforation of the membrane

doi: 10.1902/jop.2012.120371 
requires extra time and effort to repair during the surgery, or it can result in the termination of the procedure. Membrane perforation has been linked to higher postoperative complications ${ }^{8}$ and implant failures. ${ }^{9}$ It is recommended that the membrane should be elevated to the medial wall of the maxillary sinus $^{1,2}$ for the following reasons: 1) lifting the membrane to the medial wall reduces membrane tension, which is related to the occurrence of perforations; 2) a space between the elevated membrane and the medial wall (which might compromise implant placement) can be avoided; and 3) additional blood supply can be obtained from the medial wall. The sinus membrane is vascularized by branches of the posterior superior alveolar artery (PSAA) and infra-orbital artery from the lateral wall of the sinus. ${ }^{10}$ The posterior lateral nasal artery, which originates from the medial wall, adds more blood supply to the bone grafts. ${ }^{11,12}$

In light of the importance of sinus membrane management for sinus augmentation, research on sinus anatomy has emerged and received profound attention from implant surgeons. ${ }^{13}$ The distance between the branches of the PSAA and the alveolar ridge was measured from cadavers ${ }^{10}$ and cone-beam computed tomography (CBCT) scans. ${ }^{14,15}$ This artery may be encountered if it is proximate to the osteotomy site on the lateral wall, thus increasing the risk of massive hemorrhage. Sinus septa have been extensively studied with regard to their location, size, and orientation. ${ }^{14,16,17}$ The presence of a septum is associated with a higher incidence of sinus perforation. The shape of the sinus in the cross-sectional view might also be related to the occurrence of membrane perforation. ${ }^{18} \mathrm{~A}$ study ${ }^{19}$ has shown that a sharp angulation between the medial and lateral wall is associated with more perforations.

The above knowledge greatly enhances our understanding of the sinus anatomy and its variations, which can help surgeons to reduce surgical complications. The anatomy of the medial wall has never been investigated. On CBCT scans, a recess (the palatonasal recess [PNR]) between the roof of the hard palate and the lateral wall of the nasal cavity is a common finding (Fig. 1). The location and angulation of such a recess will determine the degree of difficulty in elevating the membrane on the medial wall. Therefore, the aim of this study is: 1) to evaluate the location and angulation of the PNR on CBCT scans; and 2) to identify challenging cases for performing sinus elevation according to the features of the PNR.

\section{MATERIALS AND METHODS}

This study was approved by the Institutional Review Boards of the University of Michigan
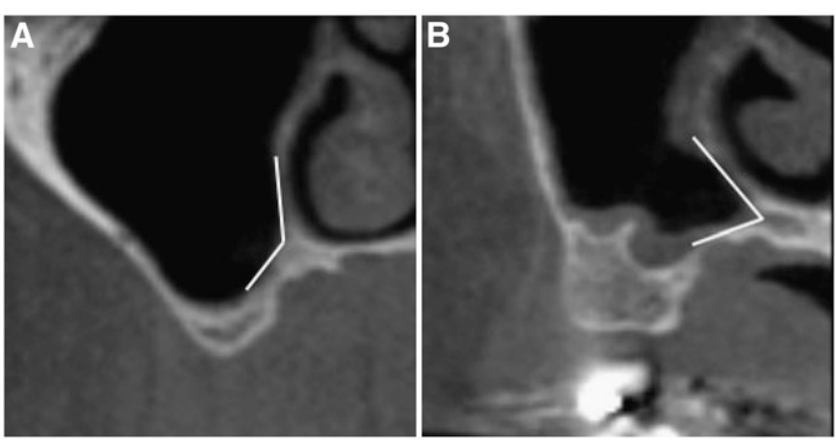

Figure $I$.

Demonstration of PNRs with an obtuse angle (A) and an acute angle $(\mathbf{B})$ on the medial wall of the maxillary sinus on $C B C T$ scans.

(HUM00049915) and was conducted from February 1 to April 30, 2012.

\section{Image Acquisition}

The scans used in the present study were selected from the CBCT database and were not specifically acquired for this project. All images were obtained with a CBCT machine ${ }^{\ddagger}$ in the Department of Periodontics and Oral Medicine, University of Michigan School of Dentistry, by board-certified oral and maxillofacial radiologists (EB and Sharon Brook) between 2005 and 2012. The imaging parameters were set at $120 \mathrm{kVp}, 18.66 \mathrm{mAs}$, scan time 20 seconds, resolution $0.4 \mathrm{~mm}$, and a field of view that varied based on the scanned region. The CBCT scans of each individual were transferred to a desktop computer equipped with an implantplanning software program. $\S$ Data were saved in the Digital Imaging and Communications in Medicine format.

\section{Inclusion Criteria}

Image screening was performed by one examiner (H-LC) using the following inclusion criteria: 1) presence of an edentulous ridge in vicinity of the maxillary sinus as a result of a missing single tooth or multiple teeth; 2) residual ridge height $(\mathrm{RH})<10$ $\mathrm{mm}$; 3) presence of adjacent or opposing teeth to the edentulous span so that the location of the edentulous ridges corresponding to the tooth site could be identified; and 4) visibility of the maxillary sinus to be measured from its floor to $\geq 15 \mathrm{~mm}$ from the alveolar crest of the edentulous ridge.

Images were excluded if: 1) they were unclear or incomplete because of scattering or other reasons; 2 ) edentulous ridge height was $>10 \mathrm{~mm}$; 3 ) location of the edentulous ridge could not be determined; 4) sinus pathology was present that made the measurement impossible; 5) the outline of the

† i-CAT, Imaging Sciences International, Hatfield, PA.

$\S$ InvivoDent, Anatomage, San Jose, CA. 
edentulous ridge could not be identified, e.g., extraction sockets; or 6 ) the sinus had received grafts or implants.

When both sinuses were eligible for the study, one sinus was randomly selected by flipping a coin. Qualified scans were reoriented so that the maxilla was bilaterally symmetric and the hard palate was parallel to the ground. The reference arch $(80 \mathrm{~mm}$ wide) was drawn at the level of the alveolar crest at the cross-sectional view, with its center set at the center of the ridge. The sagittal section that included the middle part of each missing tooth was selected. On the selected sagittal section, measurements were made by a built-in digital caliper in millimeters, including $\mathrm{RH}$ and location and angulation of the PNR. The PNR was defined as the intersection point of the two imaginary lines following the lower part of the lateral nasal wall and the palatal wall in the maxillary sinus (Fig. 1). The location of the PNR was measured from the alveolar crest in millimeters, and the angulation was measured in degrees. Two calibrated examiners (FS and AM) performed the measurements. Interexaminer and intra-examiner agreements were calculated to be 0.87 and 0.83 , respectively, with the $\kappa$ test by comparing the measurements in three randomly selected cases at two time points 3 days apart.

\section{Statistical Analyses}

The data, including the location and angulation of the PNR, were stratified by site and RH. Sites were grouped into first premolars (\#5 and \#12), second premolars (\#4 and \#13), first molars (\#3 and $\# 14$ ), and second molars (\#2 and \#15). RHs were classified as severely deficient (SvD), moderately deficient (MdD), or slightly deficient (SID) at $<4$ $\mathrm{mm}, \geq 4 \mathrm{~mm}$ to $<7 \mathrm{~mm}$, and $\geq 7 \mathrm{~mm}$ to $<10 \mathrm{~mm}$, respectively. The location and angulation of the PNR were compared among sites in the same RH group and among $\mathrm{RH}$ groups of the same sites with the one-way analysis of variance test. Post hoc examination was performed using the Dunnett test. The significance level was set at 0.05. To identify the risk group that might have a higher perforation rate due to the features of the PNR, a two-by-two table was made for each site, using the PNR location at $15 \mathrm{~mm}$ and angulation of $90^{\circ}$ as cutoff points. The $15-\mathrm{mm}$ level was chosen because that level is usually considered the upper limit for the membrane elevation; ${ }^{20}$ the $90^{\circ}$ level was chosen arbitrarily. The risk group is defined when the PNR location is $<15 \mathrm{~mm}$ and the angulation is $<90^{\circ}$. The prevalence of risk groups for each site was calculated as a percentage. Statistical analyses were conducted with commercially available software.\|

\section{RESULTS}

A total of 225 individuals (99 males and $126 \mathrm{fe}$ males; average age: 49.2 years; range: 38 to 74 years) fulfilled the inclusion criteria. Of those included, 181, 39, four, and one had one, two, three, and four missing teeth in the posterior maxilla, respectively. A total of 274 sites were evaluated, consisting of three first premolars, 40 second premolars, 147 first molars, and 84 second molars. Because of the small sample size in the first premolar sites, those data were excluded from further analyses.

The location of the PNR in relation to the alveolar crest is presented in Table 1 . In the pooled data (regardless of the $\mathrm{RH}$ ), there was a trend that the mean PNR location was gradually lower from the premolar to the molar sites. The mean PNR locations were $14.2 \pm 2.8 \mathrm{~mm}, 13.1 \pm 2.2 \mathrm{~mm}$, and $12.5 \pm 2.5 \mathrm{~mm}$ for the second premolar, first molar, and second molar sites (Fig. 2). Significant differences in the PNR location were found between the second premolar and second molar sites in the SID group $(15.2 \pm 1.8 \mathrm{~mm}$ and $13.2 \pm 2.0 \mathrm{~mm})$ and the pooled group (14.2 $\pm 2.8 \mathrm{~mm}$ and $12.5 \pm 2.5 \mathrm{~mm}$ ). The PNR location was statistically higher in the first molar sites of the SID group $(14.1 \pm 2.1 \mathrm{~mm})$ than those of the MdD $(12.6 \pm 1.8 \mathrm{~mm})$ and SvD (12.2 \pm $2.4 \mathrm{~mm}$ ) groups (Table 1).

The mean PNR angulations from the pooled data were $109.8^{\circ} \pm 25.3^{\circ}, 121.6^{\circ} \pm 22.1^{\circ}$, and $144.9^{\circ} \pm$ $23.1^{\circ}$, respectively, for the second premolar, first molar, and second molar sites (Fig. 3). The mean PNR angulation was significantly different among the three sites. When the data were stratified by RH, the PNR angulation was significantly wider in the second molar sites than in the second premolar and first molar sites (Table 2).

The two-by-two table (Table 3 ) showed that in the second premolar sites, the proportion of risk cases was higher (15\%) than in the first molar sites $(8.2 \%)$. The second molar sites had the smallest proportion of risk cases $(2.4 \%)$.

\section{DISCUSSION}

Leonardo da Vinci (1452-1519) illustrated the presence of the sinuses in the maxillary bones. He said of the sinuses, "I wish to elevate that part of the bone, the support of the cheek,... to demonstrate through the exposed opening the size and depth of the two cavities which are hidden behind it. The eye, the instrument of vision, is hidden in the cavity above, and in that below is the humor which nourishes the roots of the teeth."21 This pyramid-shaped hollow structure is located in the

\| SPSS v.19, IBM, Chicago, IL. 
Table I.

\section{Location of PNR by RH and Site}

\begin{tabular}{lcc}
\hline RH and Site & $n$ & Mean \pm SD \\
\hline SvD & & \\
Second premolar & 9 & $14.2 \pm 3.2$ \\
First molar & 30 & $12.2 \pm 2.4^{*}$ \\
Second molar & 12 & $12.2 \pm 1.9$ \\
Subtotal & 51 & $12.5 \pm 2.5$ \\
MdD & & \\
Second premolar & 11 & $13 \pm 3.6$ \\
First molar & 67 & $12.6 \pm 1.8^{\dagger}$ \\
Second molar & 36 & $12.2 \pm 3$ \\
Subtotal & 115 & $12.5 \pm 2.4$ \\
SID & & \\
Second premolar & 20 & $15.2 \pm 1.8^{\ddagger}$ \\
First molar & 50 & $14.1 \pm 2.1^{*}$ \\
Second molar & 36 & $13.2 \pm 2^{\ddagger}$ \\
Subtotal & 108 & $14.1 \pm 2.2$ \\
Total & & \\
Second premolar & 40 & $14.2 \pm 2.8^{\S}$ \\
First molar & 147 & $13.1 \pm 2.2$ \\
Second molar & 84 & $12.5 \pm 2.5^{\S}$ \\
\hline
\end{tabular}

Matching symbols denote significant difference $(P<0.05)$ between groups.

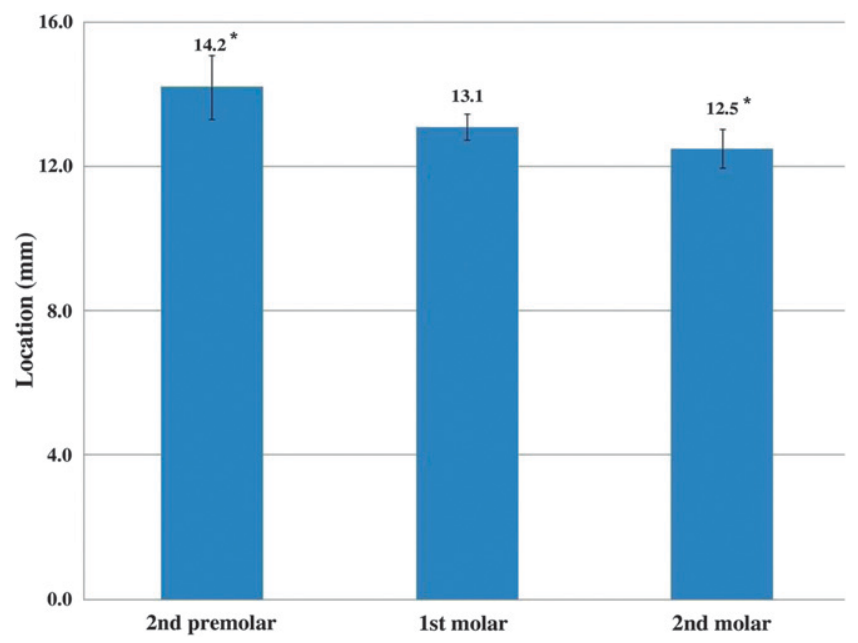

Figure 2.

Bar chart showing the location of the PNRs for the second premolar, first molar, and second molar sites. The mean location in the second molar sites was significantly closer to the alveolar crest ( $12.5 \mathrm{~mm}$ ) than in the second premolar sites ( $14.2 \mathrm{~mm}) .{ }^{*} \mathrm{P}<0.05$.

body of the maxilla, with its base at the lateral wall of the nose and apex at the zygomatic process. Although in most instances confined to the maxillary bone, it may expand into the palatine and zygomatic bones in older age. ${ }^{21}$ With the popularity of implant therapy and sinus augmentation, knowledge on the anatomy of the maxillary sinus has

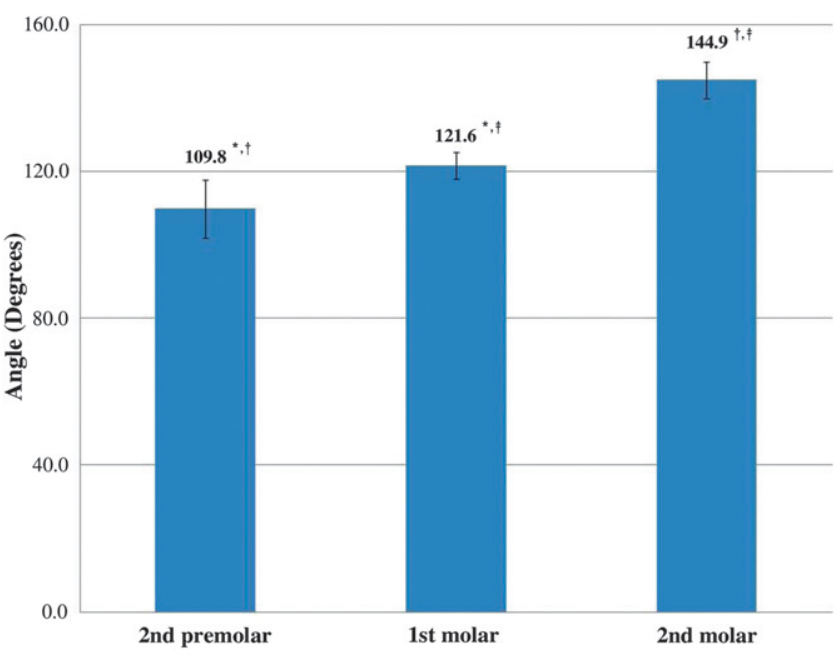

Figure 3.

Bar chart showing the angulation of the PNRs (mean \pm 2 SE, degrees). The mean angulations were $109.8^{\circ} \pm 25.3^{\circ}, 121.6^{\circ} \pm 22.1^{\circ}$, and $144.9^{\circ} \pm 23.1^{\circ}$, respectively, for the second premolar, first molar, and second molar sites. * ${ }^{\dagger}$ Significant differences $(P<0.05)$ among the three sites.

expanded tremendously. However, this study is the first to systematically evaluate the PNR in the maxillary sinus.

The PNR was studied in 274 edentulous sites via CBCT scans. CBCT scans provide accurate threedimensional reconstructed images for diagnosing and treatment planning of implant surgeries. ${ }^{22}$ The results of this study showed that the location of the PNR is closer to the crestal ridge at the molar sites than at the premolar sites. Therefore, it will be more commonly encountered at the molar sites during the SALW approach. On the other hand, its angulation is more obtuse in the molar sites, rendering an easier bypass in these regions. Taking both the location and angulation into consideration, an acute-angled PNR, which makes membrane elevation more difficult, might be present in $15 \%$ of premolar sites that are scheduled for sinus augmentation. On the contrary, only $\approx 2 \%$ of molar sites might have such an acute-angled PNR within the working field of an SALW procedure. Because the membrane is more difficult to elevate at acuteangled recesses, the amount of sinus augmentation might be limited. Additionally, the tension in the membrane might increase the incidence of perforation. The perforation may not necessarily occur at the recess but at the weakest point in the membrane. Based on the results of this study, performing sinus membrane elevation on the medial wall would be more prudent in premolar sites.

Sinus membrane perforation is the most common complication of SALW. ${ }^{6}$ The presence of a sinus septum is the most probable cause for this 
Table 2.

\section{Angulation of the Palatonasal Recess by $\mathrm{RH}$ and Site}

\begin{tabular}{|c|c|c|}
\hline $\mathrm{RH}$ and Site & $\mathrm{n}$ & Mean \pm SD \\
\hline \multicolumn{3}{|l|}{ SvD } \\
\hline Second premolar & 9 & $108.5 \pm 26.1$ * \\
\hline First molar & 30 & $115.2 \pm 19.7^{\dagger}$ \\
\hline Second molar & 12 & $149.1 \pm 19.6 * \dagger$ \\
\hline Subtotal & 51 & $\mid 22.1 \pm 25.7$ \\
\hline \multicolumn{3}{|l|}{ MdD } \\
\hline Second premolar & 11 & $115.7 \pm 21.4^{\ddagger}$ \\
\hline First molar & 67 & $122.5 \pm 24.6^{\S}$ \\
\hline Second molar & 36 & $\mid 44.1 \pm 26.7^{\neq} \S$ \\
\hline Subtotal & 115 & $128.6 \pm 27.8$ \\
\hline \multicolumn{3}{|l|}{ SID } \\
\hline Second premolar & 20 & $107.2 \pm 27.511$ \\
\hline First molar & 50 & $124.1 \pm 19.8$ \\
\hline Second molar & 36 & $144.2 \pm 16.3^{\| \mathscr{}}$ \\
\hline Subtotal & 108 & $127.4 \pm 22.8$ \\
\hline \multicolumn{3}{|l|}{ Total } \\
\hline Second premolar & 40 & $109.8 \pm 25.3^{\# * *}$ \\
\hline First molar & 147 & $121.6 \pm 22.1^{\# \dagger \dagger}$ \\
\hline Second molar & 84 & $144.9 \pm 23.1 * * \dagger \dagger$ \\
\hline
\end{tabular}

Matching symbols denote significant difference $(P<0.05)$ between groups.

Table 3.

\section{Percentage of Risk Sites in Second Premolar, First Molar, and Second Molar}

\begin{tabular}{|c|c|c|c|}
\hline \multirow[b]{2}{*}{ PNR Location* } & \multicolumn{2}{|c|}{ Angle } & \multirow[b]{2}{*}{ Total } \\
\hline & $<90^{\circ}$ & $\geq 90^{\circ}$ & \\
\hline \multicolumn{4}{|l|}{ Second premolar } \\
\hline$<15 \mathrm{~mm}$ & $6(15.0 \%)$ & 18 & 24 \\
\hline$\geq 15 \mathrm{~mm}$ & 5 & 11 & 16 \\
\hline Total & 11 & 29 & 40 \\
\hline \multicolumn{4}{|l|}{ First molar } \\
\hline$<15 \mathrm{~mm}$ & 12 (8.2\%) & 110 & 122 \\
\hline$\geq 15 \mathrm{~mm}$ & 2 & 23 & 25 \\
\hline Total & 14 & 133 & 147 \\
\hline \multicolumn{4}{|l|}{ Second molar } \\
\hline$<15 \mathrm{~mm}$ & $2(2.4 \%)$ & 66 & 68 \\
\hline$\geq 15 \mathrm{~mm}$ & 0 & 16 & 16 \\
\hline Total & 2 & 82 & 84 \\
\hline
\end{tabular}

Bold type indicates risk sites.

* Distance from alveolar crest. complication. ${ }^{14,16,17}$ Other predisposing factors include inadequate surgical experience, narrow sinuses, ${ }^{23}$ thick lateral wall, ${ }^{18}$ and thin sinus membrane. ${ }^{24}$ This study has identified another anatomic variation, the PNR, that might be associated with the occurrence of membrane perforation. Therefore, during the treatment planning phase, the location and angulation of the PNR should be carefully analyzed.

The midface forms during the fourth and seventh developmental weeks in utero, when the medial and lateral nasal prominences, the lowest part of the intermaxillary segment and the maxillary process, fuse together. ${ }^{25}$ The maxillary sinus begins its development at the 10th developmental week. It first presents as a sac with lining mucosa that is located at the deeper and anterior end of the ethmoid infundibulum, lateral to the primary uncinate process. $^{26}$ By the 15 th to 16 th week, the sinus is surrounded by a sleeve of cartilage. Shortly after, there is the first evidence of ossification on the lateral wall of the sinus, then the anterior wall between the maxillary sinus and nasolacrimal duct at the 20th week, and the posterior wall by the $21 \mathrm{st}$ week. However, at the 37th week, ossification of the medial wall is still not evident. ${ }^{25}$ Two periods of peak growth are observed for the maxillary sinus, from the 17th to the 20th week and from the 25th to the 28th week. ${ }^{27}$ The growth of the sinus follows that of the cranium, and as a result, its anteroposterior dimension increases at a greater speed than the mediolateral or supero-inferior dimensions at this stage. ${ }^{26}$ There are two other spurts of growth: from birth to the third year and between the seventh and 12th years, after which the maxillary sinus gradually reaches adult size at the age of 18 years. $^{28}$

It is not known when the PNR develops. The recess is bounded by the lateral wall of the nasal cavity and the palatal wall of the maxilla; thus the angulation of the recess might be determined when the development of these bone walls are completed. However, whether the maxillary sinus might continuously extend toward these bone walls by bone remodeling after the bone walls are fully developed is not clear. ${ }^{29}$ Regarding its location, the reference point used in this study was the alveolar crest. After the tooth is fully erupted, the distance from the recess to the alveolar crest should be stable. After the tooth is lost, depending on the amount of vertical bone loss, the distance becomes shorter. ${ }^{30}$ Shorter distances were observed in the SvD and MdD ridges than in the SID ridges in the present study, although significant differences were found only at the first molar sites (Table 1). The development of this recess should be further studied 
to understand factors that determine its position and angulation.

A number of alternatives are available with various success rates in lieu of a sinus augmentation procedure for restoration of the posterior edentulous maxilla. Short implants $(<10 \mathrm{~mm})$ might require less surgical skill and less treatment time and costs. However, the medium- and long-term prognosis is still unknown. ${ }^{31}$ Furthermore, the posterior maxilla, because of its soft bone quality, might not represent a predictable treatment modality. ${ }^{32}$ In addition, the use of tilted implants has been proposed to support fixed prostheses for rehabilitation of limited-height edentulous ridges. Results have shown that combining tilted and straight implants for supporting fixed prostheses can be considered a viable treatment modality because of the high survival rate and low rate of prosthetic complications. ${ }^{33}$ Zygomatic implants might be another option, although they are not as popular as regular implants. ${ }^{34}$ Cantilever prostheses could also function well, if provided with thorough biomechanical considerations. Nonetheless, cantilever prostheses might incur higher rates of prosthetic complications, such as abutment loosening and denture fracture, if they are $>12 \mathrm{~mm}$ in the maxilla. ${ }^{35}$ When planning a sinus augmentation surgery, other alternatives should be considered and discussed with the patient, especially when the risk of performing the surgery might outweigh its benefits.

Future studies should focus on studying anatomic landmarks that are correlated with the location and angulation of the PNR. Considering the close relationships among the maxillary sinus, palate, alveolar process, and nasal cavity from a spatial and embryologic point of view, possible correlations might exist among the following measurements: 1) the palatal vault depth and the distance between the PNR and the alveolar crest; 2) the shape of the palatal vault and the angulation of the PNR; and 3) the width of the nasal cavity and the angulation of the PNR. Understanding these possible correlations can help surgeons to predict potentially difficult cases that are related to the PNR during diagnosis and treatment planning of SALW surgery.

\section{CONCLUSIONS}

This CBCT study evaluates the location and angulation of the PNR in the maxillary sinus from 274 edentulous sites. At the second premolar sites, 15\% of the recesses are $<90^{\circ}$ and $\leq 15 \mathrm{~mm}$ from the alveolar crest, compared to $8.2 \%$ and $2.4 \%$ at the first and second molar sites, respectively. Sharpangled recesses might complicate sinus membrane elevation on the medial wall and increase the occurrence of membrane perforation. Therefore, this anatomic structure should be considered when planning the SALW approach.

\section{ACKNOWLEDGMENTS}

The authors thank Dr. Sharon Brook, a radiologist now retired from the University of Michigan, for providing CBCT scans. The authors do not have any financial interests, either directly or indirectly, in the products or information listed in the paper. This paper was partially supported by the University of Michigan Periodontal Graduate Student Research Fund. The authors report no conflicts of interest related to this study.

\section{REFERENCES}

1. Boyne PJ, James RA. Grafting of the maxillary sinus floor with autogenous marrow and bone. J Oral Surg 1980;38:613-616.

2. Tatum H Jr. Maxillary and sinus implant reconstructions. Dent Clin North Am 1986;30:207-229.

3. Vercellotti T, De Paoli S, Nevins M. The piezoelectric bony window osteotomy and sinus membrane elevation: Introduction of a new technique for simplification of the sinus augmentation procedure. Int $J$ Periodontics Restorative Dent 2001;21:561-567.

4. Chan HL, Oh TJ, Fu JH, Benavides E, Avila-Ortiz G, Wang HL. Sinus augmentation via transcrestal approach: A comparison between the balloon and osteotome technique in a cadaver study [published online ahead of print June 21, 2012]. Clin Oral Implants Res. doi:10.1111/j.1600-0501.2012.02506.x.

5. Triplett RG, Nevins M, Marx RE, et al. Pivotal, randomized, parallel evaluation of recombinant human bone morphogenetic protein-2/absorbable collagen sponge and autogenous bone graft for maxillary sinus floor augmentation. J Oral Maxillofac Surg 2009;67:19471960.

6. Pjetursson BE, Tan WC, Zwahlen M, Lang NP. A systematic review of the success of sinus floor elevation and survival of implants inserted in combination with sinus floor elevation. J Clin Periodontol 2008; 35 (Suppl. 8)216-240.

7. Tonetti MS, Hämmerle CH; European Workshop on Periodontology Group C. Advances in bone augmentation to enable dental implant placement: Consensus Report of the Sixth European Workshop on Periodontology. J Clin Periodontol 2008; 35 (Suppl. 8)168-172.

8. Schwartz-Arad D, Herzberg R, Dolev E. The prevalence of surgical complications of the sinus graft procedure and their impact on implant survival. J Periodontol 2004;75:511-516.

9. Cho-Lee GY, Naval-Gias L, Castrejon-Castrejon S, et al. A 12-year retrospective analytic study of the implant survival rate in 177 consecutive maxillary sinus augmentation procedures. Int J Oral Maxillofac Implants 2010;25:1019-1027.

10. Solar P, Geyerhofer U, Traxler H, Windisch A, Ulm C, Watzek G. Blood supply to the maxillary sinus relevant to sinus floor elevation procedures. Clin Oral Implants Res 1999;10:34-44. 
11. Flanagan D. Arterial supply of maxillary sinus and potential for bleeding complication during lateral approach sinus elevation. Implant Dent 2005;14:336338.

12. Rosano G, Taschieri S, Gaudy JF, Del Fabbro M. Maxillary sinus vascularization: A cadaveric study. J Craniofac Surg 2009;20:940-943.

13. Chan HL, Wang HL. Sinus pathology and anatomy in relation to complications in lateral window sinus augmentation. Implant Dent 2011;20:406-412.

14. Güncü GN, Yildirim YD, Wang HL, Tözüm TF. Location of posterior superior alveolar artery and evaluation of maxillary sinus anatomy with computerized tomography: A clinical study. Clin Oral Implants Res 2011;22: 1164-1167.

15. Elian N, Wallace S, Cho SC, Jalbout ZN, Froum S. Distribution of the maxillary artery as it relates to sinus floor augmentation. Int J Oral Maxillofac Implants 2005;20:784-787.

16. Kim MJ, Jung UW, Kim CS, et al. Maxillary sinus septa: Prevalence, height, location, and morphology. A reformatted computed tomography scan analysis. J Periodontol 2006;77:903-908.

17. Krennmair G, Ulm CW, Lugmayr H, Solar P. The incidence, location, and height of maxillary sinus septa in the edentulous and dentate maxilla. J Oral Maxillofac Surg 1999;57:667-671; discussion 671-662.

18. Velloso GR, Vidigal GM Jr., de Freitas MM, Garcia de Brito OF, Manso MC, Groisman M. Tridimensional analysis of maxillary sinus anatomy related to sinus lift procedure. Implant Dent 2006;15:192-196.

19. Cho SC, Wallace SS, Froum SJ, Tarnow DP. Influence of anatomy on Schneiderian membrane perforations during sinus elevation surgery: Three-dimensional analysis. Pract Proced Aesthet Dent 2001;13:160-163.

20. Wang HL, Katranji A. ABC sinus augmentation classification. Int J Periodontics Restorative Dent 2008;28: 383-389.

21. Sperber GH. Applied anatomy of the maxillary sinus. $J$ Can Dent Assoc 1980;46:381-386.

22. Chan HL, Misch K, Wang HL. Dental imaging in implant treatment planning. Implant Dent 2010;19:288-298.

23. Katranji A, Fotek P, Wang HL. Sinus augmentation complications: Etiology and treatment. Implant Dent 2008; 17:339-349.

24. Pommer B, Unger E, Sütö D, Hack N, Watzek G. Mechanical properties of the Schneiderian membrane in vitro. Clin Oral Implants Res 2009;20:633-637.

25. Neskey D, Eloy JA, Casiano RR. Nasal, septal, and turbinate anatomy and embryology. Otolaryngol Clin North Am 2009;42:193-205, vii.
26. Nuñez-Castruita A, López-Serna N, Guzmán-López S. Prenatal development of the maxillary sinus: A perspective for paranasal sinus surgery. Otolaryngol Head Neck Surg 2012;146:997-1003.

27. Lawson W, Patel ZM, Lin FY. The development and pathologic processes that influence maxillary sinus pneumatization. Anat Rec (Hoboken) 2008;291: 1554-1563.

28. Adibelli $\mathrm{ZH}$, Songu $M$, Adibelli $H$. Paranasal sinus development in children: A magnetic resonance imaging analysis. Am J Rhinol Allergy 2011;25:30-35.

29. Alberti PW. Applied surgical anatomy of the maxillary sinus. Otolaryngol Clin North Am 1976;9:3-20.

30. Tan WL, Wong TL, Wong MC, Lang NP. A systematic review of post-extractional alveolar hard and soft tissue dimensional changes in humans. Clin Oral Implants Res 2012;23 (Suppl. 5):1-21.

31. Felice P, Soardi E, Pellegrino G, et al. Treatment of the atrophic edentulous maxilla: Short implants versus bone augmentation for placing longer implants. Fivemonth post-loading results of a pilot randomised controlled trial. Eur J Oral Implantology 2011;4:191 202.

32. Monje A, Chan HL, Fu JH, Suarez F, Galindo-Moreno P, Wang HL. Are Short Dental Implants $(<10 \mathrm{~mm})$ Effective? A Meta-Analysis on Prospective Clinical Trials [published ahead of print August 23, 2012]. J Periodontol. doi:10.1902/jop.2012.120328.

33. Monje A, Chan HL, Suarez F, Galindo-Moreno P, Wang HL. Marginal bone loss around tilted implants in comparison to straight implants: A meta-analysis. Int J Oral Maxillofac Implants 2012;27:1576-1583.

34. Davo R, Pons O, Rojas J, Carpio E. Immediate function of four zygomatic implants: A 1-year report of a prospective study. Eur J Oral Implantology 2010;3:323334.

35. Shackleton JL, Carr L, Slabbert JC, Becker PJ. Survival of fixed implant-supported prostheses related to cantilever lengths. J Prosthet Dent 1994;71:23-26.

Correspondence: Dr. Hom-Lay Wang, Department of Periodontics and Oral Medicine, University of Michigan School of Dentistry, 1011 North University Avenue, Ann Arbor, MI 48109-1078. Tel: 734/763-3383; Fax: 734/9360374; e-mail: homlay@umich.edu.

Submitted June 14, 2012; accepted for publication September 1, 2012. 